\title{
AÇÃO PROTETORA, RESIDUAL, CURATIVA E ANTI ESPORULANTE DE FUNGICIDAS NO CONTROLE DA REQUEIMA E DA PINTA PRETA DA BATATA EM CONDIÇÕES CONTROLADAS
}

\author{
J.G. Töfoli ${ }^{1}$, P.C.T. Melo ${ }^{2}$, R.J. Domingues ${ }^{1}$ \\ ${ }^{1}$ Instituto Biológico, Centro de Pesquisa e Desenvolvimento deSanidade Vegetal, Av. Cons. Rodrigues Alves, \\ 1252, CEP 04014-002, São Paulo, SP. Brasil. E-mail: tofoli@biologico.sp.gov.br
}

\section{RESUMO}

\begin{abstract}
A ação de fungicidas em diferentes fases do processo infeccioso da requeima (Phytophthora infestans) e da pinta preta (Alternaria solani) da batata foi estudada em condições de casa-de-vegetação e laboratório. A ação protetora e residual foi avaliada através de inoculações realizadas com os respectivos patógenos a 1, 3, 6, 9, 12 e 15 dias após a pulverização (DAP). Para avaliar a ação curativa, os fungicidas foram aplicados 1,12, 24 e 48 horas após a inoculação (HAI) para requeima e 1, 24, 48 e 72 HAI para a pinta preta. As ações preventiva, residual e curativa foram avaliadas com base na porcentagem de área foliar afetada e a ação anti-esporulante através da contagem de esporângios e conídios. Todos os fungicidas promoveram elevada proteção contra a requeima e a pinta preta. Os fungicidas sistêmicos apresentaram redução de controle a partir dos $12 \mathrm{DAP}$, enquanto que os fungicidas translaminares e mesostêmicos a partir dos $9 \mathrm{DAP}$. Quanto à ação curativa e anti esporulante, destacaram-se principalmente os fungicidas sistêmicos aplicados até as 24 horas após a inoculação (HAI). Fungicidas translaminares e mesostêmicos foram capazes de inibir a requeima quando aplicados até $12 \mathrm{HAI}$. Os fungicidas de contato destacaram-se apenas para ação protetora.
\end{abstract}

PALAVRAS-CHAVE: Solanum tuberosum, Phytophthora infestans, Alternaria solani, controle químico.

\section{ABSTRACT}

PROTECTIVE, RESIDUAL, CURATIVE AND ANTISPORULATING ACTION OF FUNGICIDES IN THE CONTROL OF EARLY AND LATE BLIGHT OF POTATOES UNDER CONTROLLED CONDITIONS. The action of fungicides at different phases of the infectious process of late blight (Phytophthora infestans) and early blight (Alternaria solani) of potatoes was studied under greenhouse and laboratory conditions. Protective and residual action was evaluated through inoculations undertaken with the respective pathogens $1,3,6,9,12$ and 15 days after application. To evaluate the curative action against late blight, fungicides were applied 1, 12, 24 and 48 hours after inoculation, and against early blight 1, 24, and 48 hours after inoculation. Protective, residual and curative actions were evaluated according to the percentage of the foliar area affected, while the antisporulating action was assessed through sporangia and conidia counting. All the fungicides, regardless of their mode of action, provided high protection against late and early blight. Systemic fungicides showed a reduction of control starting at 12 days after application, while translaminar and mesostemic fungicides showed this reduction starting at 9 days after application. In regard to curative and antisporulating actions, systemic fungicides applied until 24 hours after inoculation were especially outstanding. Translaminar and mesostemic fungicides could inhibit late blight when applied until 12 hours after inoculation. Contact fungicides stood out in preventive action only.

KEY WORDS: Solanum tuberosum, Phytophthora infestans, Alternaria solani, chemical control.

\section{INTRODUÇÃO}

A requeima (Phytophthtora infestans) e a pinta preta (Alternaria solani) são as mais importantes e destrutivas doenças foliares da cultura da batata, no
Brasil e no mundo. O uso de fungicidas representa uma medida de controle eficiente e necessária em situações onde coexistem cultivares suscetíveis, condições meteorológicas favoráveis e cultivos sucessivos dessa solanácea.

${ }^{2}$ Universidade de São Paulo, Escola Superior de Agricultura "Luiz de Queiroz", Departamento de Produção Vegetal, Piracicaba, SP, Brasil. 
A constante evolução técnico-científica dos fungicidas agrícolas permitiu o desenvolvimento de produtos com diversos modos de ação na planta e nas diferentes fases do processo infeccioso (Agrios, 2005; RusSEL, 2005). Em relaçãoà planta, os fungicidas podem ser classificados em produtos de contato, mesostêmicos, translaminares e sistêmicos (HEwIT, 1998; ReIs et al., 2007). Os fungicidas de contato caracterizam-se por formar uma película protetora na superfície da planta, que impede a penetração do patógeno. Os fungicidas mesostêmicos apresentam alta afinidade com a camada cerosa superficial das folhas, podendo se redistribuir na fase de vapor ou ser absorvido pelo tecido, sem, no entanto, apresentar nenhum movimento. Os fungicidas sistêmicos são aqueles que podem se movimentar na planta através de vasos condutores podendo atingir locais distantes do local depositado, enquanto que os translaminares distribuem-se de forma limitada nos tecidos (AzEvEDo, 2007; ReIs et al., 2007). Quanto ao processo infeccioso, os fungicidas podem apresentar ação protetora, curativa e anti esporulante. A ação protetora se expressa quando esse é aplicado antes do patógeno infectar os tecidos da planta. Fatores como volume, número e intervalo de aplicações e a tecnologia de aplicação utilizada podem inferir diretamente sobre o potencial de proteção de um fungicida (TöFoli, 2006). A ação curativa consiste na capacidade do fungicida em limitar o desenvolvimento do patógeno no interior dos tecidos, quando aplicado no período latente, ou seja, no intervalo entre a penetração e o aparecimento dos primeiros sintomas. Entre os fatores que podem influenciar a ação curativa de um fungicida destacam-se a suscetibilidade do hospedeiro, a pressão de doença, as condições meteorológicas e o momento da aplicação. (GENET et al., 2000; AzEvedo, 2007; HuggenBerger; KNAUf-BeIter, 2007). A atividade anti esporulante baseia-se na característica do fungicida em limitar a reprodução ou inviabilizar as estruturas reprodutivas do patógeno. A ação residual, por sua vez, refere-se ao período de proteção proporcionado pelo produto após a sua aplicação e pode variar em função da estabilidade da molécula, tenacidade, crescimento da planta e ocorrência de intempéries (REIs et al., 2007; BAIN, 2009).

Níveis diferenciados de ação protetora, residual, curativa e anti esporulante de fungicidas no controle da requeima têm sido observados para mandipropamida (HERMANN et al., 2005; HugGenBERGER; Knauf-BeIter, 2007; ThOMPSON; CoOKe, 2009), fluopicolide (TAFFOREAU et al., 2006; LATORSE et al., 2007), bentiavalicarbe (MIYAKE et al., 2005), mefenoxam (BodKer; Nielsen, 2001; BodKer; Nielsen 2002; SinGH; Pundhir, 2004), fenamidona (TAFForeau et al., 2009), dimetomorfe (JoHNSON et al., 2000), zoxamida (BRADSHAW; SCHEPERS, 2001), propamocarbe (JOHNSON et al., 2000); BodKer; Nielsen, 2001; BodKer; Nielsen 2002), cimoxanil (GENET et al., 2000); ametoctradina (Gold et al., 2009; ReIMANn et al., 2010); e tebuconazol (ForCelini, 1994; Tofoli et al., 2005), difenoconazol (BOUWMAN; RIJKERS, 2004; TOFOLI etal., 2005; MANTECóN, 2009; HorsFIELD et al., 2010), azoxistrobina (BouwMAN; RIJKERS, 2004; TOFOLI et al., 2005; HORSFIELD et al., 2010) e boscalida (ToFoli et al., 2005; HoRsField et al., 2010) para a pinta preta da batata.

O conhecimento detalhado do perfil técnico de um fungicida permite que esse seja posicionado corretamenteem programas de manejoe, assim, expresse de forma completa o seu potencial de controle. $\mathrm{O}$ objetivo desse trabalho foi avaliar a ação protetora, residual, curativa e anti esporulante de diferentes grupos fungicidas no controle da requeima e da pinta preta da batata, em condições de casa de vegetação e laboratório.

\section{MATERIAL E MÉTODOS}

Para avaliar a ação protetora e residual de fungicidas no controle da requeima, plantas de batata da cv. Ágata, cultivadas em vasos, foram pulverizadas com os fungicidas (\% de ingrediente ativo - $\mathrm{kg}$ ou L de produto formulado - p.f. ha $^{-1}$ ): piraclostrobina + metiram $(5+50-1,5)$; dimetomorfe + ametoctradina $(22,5+30-1,0)$; dimetomorfe + mancozebe $(50+80-0,45+3,0)$; dimetomorfe + clorotalonil $(50+82,5-0,45+1,5)$; cimoxanil + mancozebe $(8+$ $64-2,0)$; cimoxanil + zoxamida $(31,1+31,1-0,4)$; mefenoxam + mancozebe $(4+64-2,5)$; mefenoxam + clorotalonil $(6,75+67,5-1,5)$; bentiavalicarbe + fluazinam $(10+25-0,7)$; mandipropamida $(25$ $0,4)$; mandipropamida + clorotalonil $(4+40-2,0)$; famoxadona + cimoxanil $(22,5+33-0,6)$; fenamidona (50 - 0,3); propamocarbe $(72,2-1,25)$; fenamidona + propamocarbe $(7,5+37,5-2,0)$; fluopicolide + propamocarbe $(6,25+62,5-2,0)$; ametoctradina + metiram (12 + 44 - 2,0); ciazofamida $(40-0,25)$; fluazinam $(50-1,0)$, mancozebe $(80-3,0)$ e clorotalonil $(82,5-1,5)$ (Experimento 1). A inoculação foi realizada em folhas, previamente marcadas, com uma suspensão de $10^{5}$ esporângios. $\mathrm{mL}^{-1}$ nos intervalos de 1, 3, 6, 9, 12 e 15 dias após a pulverização (DAP) e mantidas em câmera úmida por 36 horas a $20 \pm 2^{\circ} \mathrm{C}$. A severidade da requeima foi avaliada através de uma escala de notas de 1 a 5, adaptada da escala diagramática proposta por JAMES (1971), onde: 1 - ausência de sintomas; 2 - traços a $10 \%$; $3-10,1$ a $25 \%$; $4-25,1$ a $50 \%$ e 5 - acima de $50 \%$ de área foliar lesionada. A avaliação foi realizada seis dias após a inoculação, avaliando-se 12 folhas marcadas por parcela.

Para avaliar a ação curativa e a atividade anti esporulante de fungicidas no controle da requeima 
da batata, folhas previamente marcadas de plantas de batata Cv. Ágata foram inoculadas com uma suspensão de $10^{5}$ esporângios. $\mathrm{mL}^{-1} \mathrm{e}$ submetidas à câmara úmida por 36 horas a $20 \pm 2^{\circ} \mathrm{C}$ (Experimento 2). Os fungicidas (\% de ingrediente ativo - $\mathrm{kg}$ ou L de p.f. ha- $\left.{ }^{-1}\right)$ : piraclostrobina + metiram $(5+50-$ $1,5)$, dimetomorfe + ametoctradina $(22,5+30-1,0)$, dimetomorfe + mancozebe $(50+80-0,45+3,0)$, dimetomorfe + clorotalonil $(50+82,5-0,45+1,5)$, cimoxanil + mancozebe $(8+64-2,0)$, cimoxanil + zoxamida $(31,1+31,1-0,4)$, mefenoxam + mancozebe $(4+64-2,5)$, mefenoxam + clorotalonil $(6,75$ $+67,5-1,5)$, bentiavalicarbe + fluazinam $(10+25$ $-0,7)$, mandipropamida $(25-0,4)$, mandipropamida + clorotalonil $(4+40-2,0)$, famoxadona + cimoxanil $(22,5+33-0,6)$, fenamidona $(50-0,3)$, propamocarbe $(72,2-1,25)$, fenamidona + propamocarbe $(7,5$ $+37,5-2,0)$, fluopicolide + propamocarbe $(6,25+$ 62,5 - 2,0), ciazofamida $(40-0,25)$, fluazinam (50 $1,0)$ foram aplicados 1, 12, 24, 36 e 48 horas após a inoculação (HAI). A avaliação da severidade foi realizada com base na escala de notas adotada no experimento 1. Para avaliar a atividade anti esporulante, 12 folíolos com sintomas, por parcela, foram coletados e mantidos em câmara úmida a 18 $\pm 1^{\circ} \mathrm{C}$ por 24 horas. Após a maceração desses em $30 \mathrm{~mL}$ de água destilada estéril, determinou-se a quantidade de esporângios de $P$. infestans em microscópio óptico, através de contagens realizadas em 10 lâminas/parcela, preparadas com $10 \mu \mathrm{L}$ da suspensão.

A ação protetora e residual de fungicidas no controle da pinta preta da batata foi avaliada em um experimento onde plantas de batata da Cv. Monalisa foram pulverizadas com os fungicidas (\% de i.a. - $\mathrm{kg}$ ou L de p.f. ha-1 $)$ : picoxistrobina (25 - 0,25), azoxistrobina $(50-0,08)$, azoxistrobina + difenoconazol $(20+$ $12,5-0,75)$, piraclostrobina + metiram $(5+50-1,5)$, piraclostrobina + metconazol $(13+8-0,6)$, trifloxistrobina + tebuconazol $(20+10-0,75)$, tebuconazol $(20$ - 1,0), difenoconazol $(25-0,3)$, metconazol $(0,9-1,0)$, flutriafol $(12,5-0,75)$, boscalida $(50-0,10)$, boscalida + piraclostrobina $(20+10-0,25)$, ciprodinil $(75-0,25)$, pirimetanil (30 - 1,0), famoxadona + mancozebe $(6,25$ $+62,5-1,2)$, iprodiona + pirimetanil $(15+25-0,3+$ $0,5)$, fluazinam $(50-1,0)$, iprodiona $(50-1,0)$, clorotalonil $(82,5-1,5)$ e mancozebe $(80-3,0)$ (Experimento $3)$. Folhas marcadas foram inoculadas com uma suspensão de $10^{4}$ conídios.mL $\mathrm{mL}^{-1}$ de $A$. solani aos 1,3 , $6,9,12,15$ dias após a pulverização (DAP), sendo as plantas mantidas em câmera úmida por 24 horas a $25 \pm 2^{\circ} \mathrm{C}$. A severidade da pinta preta foi avaliada através de uma escala de notas de 1 a 6 , adaptada de REIFSCHNEIDER et al. (1984) onde: 1 - ausência de sintomas; 2 - traços a 2,4 \%; 3 - 2,5 a 11,9 \%; 4 - 12,9 a $24,9 \% ; 5-25,0$ a $50,0 \%$ e 6 - acima de $50 \%$ de área foliar lesionada. A avaliação foi realizada sete dias após a inoculação, avaliando-se 12 folhas marcadas por parcela.

Para avaliar a ação curativa e anti esporulante de fungicidas no controle da pinta preta, folhas previamente marcadas em plantas de batata $\mathrm{Cv}$. Monalisa, com aproximadamente $28 \mathrm{~cm}$ de altura, foram inoculadas com uma suspensão de $10^{4}$ conídios. $\mathrm{mL}^{-1}$ de $A$. solani e submetidas à câmara úmida por 24 horas a $25 \pm 2^{\circ} \mathrm{C}$ (Experimento 4). A pulverização dos fungicidas (\% de ingrediente ativo - $\mathrm{kg}$ de p.f. $\left.\mathrm{ha}^{-1}\right)$ : picoxistrobina $(25-0,25)$, azoxistrobina $(50-$ $0,08)$, azoxistrobina + difenoconazol $(20+12,5-0,75)$, piraclostrobina + metiram $(5+50-1,5)$, piraclostrobina + metconazol $(13+8-0,6)$, trifloxistrobina + tebuconazol $(20+10-0,75)$, tebuconazol $(20-1,0)$, difenoconazol (25 - 0,3), metconazol $(0,9-1,0)$, flutriafol $(12,5-0,75)$, boscalida $(50-0,1)$, boscalida + piraclostrobina $(20+10-0,25)$, ciprodinil $(75-0,25)$, pirimetanil $(30-1,0)$, famoxadona + mancozebe $(6,25$ $+62,5-1,2)$, iprodiona + pirimetanil $(15+25-0,3$ $+0,5)$, fluazinam $(50-1,0)$ e iprodiona $(50-1,0)$ foi realizada nos intervalos de 24,48 e 72 horas após a inoculação (HAI). A avaliação da severidade foi realizada com a escala de notas adotada no experimento 3. A quantificação de conídios de A. solani foi realizada segundo metodologia adotada no experimento 2. Nesse caso, os folíolos foram mantidos em câmara úmida a $25 \pm 1^{\circ} \mathrm{C}$ por 24 horas e fotoperíodo de 12 horas.

Nos quatro experimentos, as plantas de batata foram cultivadas em vasos plásticos (PT15), contento substrato à base de casca de pinus, vermiculita expandida, turfa processada eenriquecido com nutrientes, especialmente desenvolvido para solanáceas. Em cada vaso foram plantados dois tubérculos brotados originando plantas com 2 a 3 hastes. As plantas foram conduzidas para evitar o acamamento. A aplicação dos fungicidas foi realizada com um pulverizador costal pressurizado a $\mathrm{CO}_{2}$, equipado com uma lança de ponta única (bico cônico do tipo TXKV26), pressão constante de 3 Bar, volume de aplicação de $300 \mathrm{~L} \mathrm{ha}^{-1}$ e distância de aplicação entre a ponteira e o alvo de aproximadamente $0,5 \mathrm{~m}$.

O delineamento experimental utilizado foi o de blocos ao acaso, sendo cada parcela composta por três vasos. Para a análise estatística foi adotado um esquema fatorial e as médias foram comparadas pelo teste de Tukey a $5 \%$ de probabilidade.

\section{RESULTADOS E DISCUSSÃO}

No experimento 1 , todos os fungicidas testados apresentaram elevada ação protetora no controle da requeima da batata. A primeira redução significativa de controle foi observada nas parcelas tratadas com mancozebe aos 6 DAP (Tabela 1). Nos 
intervalos de 9 e $12 \mathrm{DAP}$, os maiores níveis de controle da requeima foram obtidos com mefenoxam + clorotalonil, mandipropamida + clorotalonil, fluopicolide + propamocarbe, bentiavalicarbe + fluazinam, mefenoxam + mancozebe, fenamidona + propamocarbe, mandipropamida, dimetomorfe + ametoctradina, dimetomorfe + clorotalonil, propamocarbe, famoxadona + cimoxanil, fenamidona e cimoxanil + zoxamida. Piraclostrobina + metiram, dimetomorfe + mancozebe, fluazinam, ametoctradina + metiram, ciazofamida e cimoxanil + mancozebe promoveram controle intermediário, enquanto que clorotalonil e mancozebe foram os menos efetivos. Aos 15 DAP, mandipropamida + clorotalonil, fluopicolide + propamocarbe, mefenoxam + clorotalonil, mandipropamida, dimetomorfe + ametoctradina, fenamidona + propamocarbe e propamocarbe foram os tratamentos mais eficientes. Neste intervalo, as parcelas tratadas com mancozebe não diferiram da testemunha. Verificou-se significativa redução no controle da requeima em função do aumento do período entre a aplicação do fungicida e a inoculação do patógeno. Clorotalonil e mancozebe tiveram seu potencial de controle reduzido a partir dos $6 \mathrm{DAP}$, enquanto que fluazinam, fenamidona, dimetomorfe + mancozebe, cimoxanil + mancozebe, ciazofamida e piraclostrobina + metiram aos 9 DAP. Os demais fungicidas apresentaram redução significativa de controle a partir dos 12 DAP.

No experimento 2, a ação curativa e anti esporulante de fungicidas no controle da requeima foi decrescente em função do aumento do período entre a inoculação e aplicação dos fungicidas (Tabela 2). No intervalo de aplicação realizado 1 hora após a inoculação (HAI), traços da requeima foram observados apenas nas parcelas tratadas com ciazofamida e fluazinam. No intervalo de $12 \mathrm{HAI}$, mefenoxam + clorotalonil, mefenoxam + mancozebe, fenamidona + propamocarbe, fluopicolide + propamocarbe, bentiavalicarbe + fluazinam, propamocarbe e famoxadona + cimoxanil promoveram os maiores níveis de controle curativo da requeima. Mandipropamida + clorotalonil mandipropamida, cimoxanil + mancozebe, cimoxanil + zoxamida, dimetomorfe + ametoctradina, fenamidona dimetomorfe + clorotalonil, piraclostrobina + metiram e dimetomorfe + mancozebe apresentaram desempenho intermediário, enquanto que fluazinam e ciazofamida foram os menos efetivos. Nos intervalos de 24, 36 e $48 \mathrm{HAI}$, as menores severidades foram obtidas com mefenoxam+clorotalonil, bentiavalicarbe + fluazinam, mefenoxam + mancozebe, fenamidona + propamocarbe fluopicolide + propamocarbe e propamocarbe. Famoxadona + cimoxanil foi semelhante aos melhores tratamentos quando aplicado 24 HAI, porém, não diferiu da testemunha no último intervalo. Mandipropamida e mandipropamida + clorotalonil proporcionaram controle intermediário em todos os períodos. Dimetomorfe + mancozebe, dimetomorfe + clorotalonil, cimoxanil + zoxamida, piraclostrobina + metiram, cimoxanil + mancozebe, famoxadona + cimoxanil e fenamidona apresentaram comportamento intermediário nos intervalos de 24 e 36 HAI e semelhante à testemunha no último. Ciazofamida e fluazinam não diferiram da testemunha em nenhum desses intervalos. Os melhores níveis de ação curativa foram obtidos com os fungicidas mefenoxam + clorotalonil, menofexam + mancozebe, propamocarbe, fenamidona + propamocarbe, fluopicolide + propamocarbe e dimetomorfe + ametoctradina aplicados até 24 HAI. Os demais tratamentos reduziram a requeima apenas no intervalo de $12 \mathrm{HAI}$. Os melhores níveis de ação anti esporulante sobre $P$. infestans foram observados quando os fungicidas foram aplicados 12 e 24 horas após a inoculação (Tabela 3). Nesses intervalos fluazinam e ciazofamida foram os menos eficientes, sendo semelhantes entre si e inferiores aos demais. Nas aplicações realizadas 36 e $48 \mathrm{HAI}$, as menores quantidades de esporângios foram obtidas com fluopicolide + propamocarbe, fenamidona + propamocarbe, mandipropamida, mandipropamida + clorotalonil, mefenoxam + clorotalonil, mefenoxam + mancozebe, dimetomorfe + ametoctradina, enquanto que fluazinam e ciazofamida foram os menos efetivos, sendo fluazinam semelhante à testemunha e inferior a ciazofamida, no intervalo de $48 \mathrm{HAI}$. Os demais tratamentos apresentaram comportamento intermediário.

No experimento 3, traços da doença foram observados nas plantas tratadas com clorotalonil e mancozebe a partir dos 6 DAP (Tabela 4). Nos intervalos de 9, 12 e $15 \mathrm{DAP}$ as parcelas tratadas com azoxistrobina + difenoconazol, trifloxistrobina + tebuconazol, piraclostrobina + metconazol, boscalida + piraclostrobina, famoxadona + mancozebe e iprodiona + pirimetanil apresentaram os menores níveis da doença. Picoxistrobina, azoxistrobina, pirimetanil, piraclostrobina + metiram, metconazol, ciprodinil, difenoconazol, tebuconazol, boscalida apresentaram comportamento intermediário, enquanto que iprodiona, clorotalonil, fluazinam e mancozebe foram os menos efetivos. Houve significativa redução no controle da pinta preta em função do aumento do período entre a pulverização e a inoculação. Os fungicidas azoxistrobina + difenoconazol, trifloxistrobina + tebuconazol, boscalida + piraclostrobina, piraclostrobina + metconazol e famoxadona + mancozebe apresentaram redução no controle a partir dos 12 DAP. Mancozebe e os demais tratamentos tiveram seus potenciais de controle reduzidos a partir dos 6 e 9 DAP, respectivamente. 


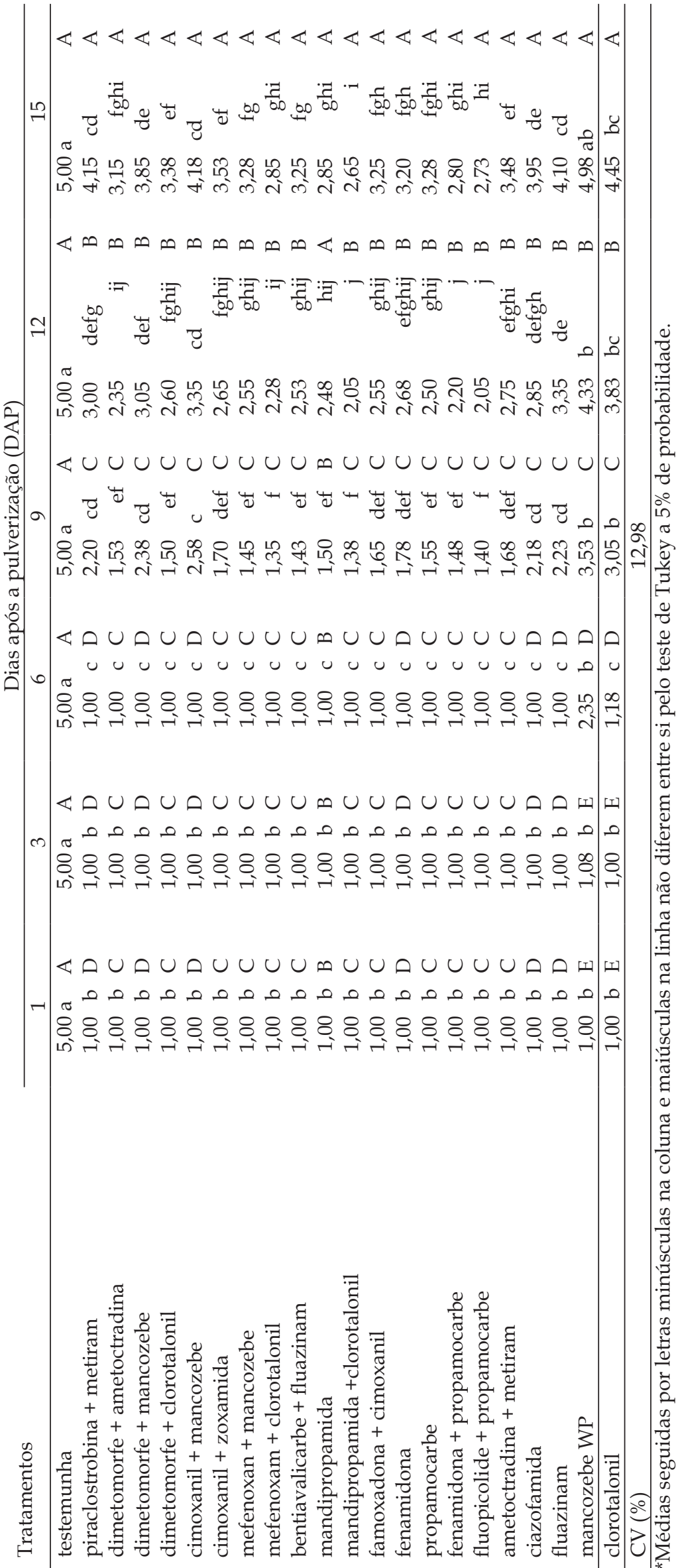


Tabela 2 - Severidade da requeima em plantas de batata do cultivar Ágata inoculadas com Phytophthora infestans e tratadas com fungicidas 1, 12, 24, 36 e 48 horas após a inoculação (HAI).

\begin{tabular}{|c|c|c|c|c|c|c|c|c|c|c|c|c|}
\hline \multirow{3}{*}{$\begin{array}{l}\text { Tratamentos } \\
\text { testemunha }\end{array}$} & \multicolumn{12}{|c|}{ Horas após a inoculação (HAI) } \\
\hline & \multicolumn{2}{|l|}{1} & \multicolumn{3}{|c|}{12} & \multicolumn{2}{|l|}{24} & \multicolumn{3}{|c|}{36} & \multicolumn{2}{|l|}{48} \\
\hline & $5,00 \mathrm{a}$ & A & $5,00 \mathrm{a}$ & & A & $5,00 \mathrm{a}$ & A & 5,00 & & A & $5,00 \mathrm{a}$ & A \\
\hline piraclostrobina + metiram & $1,00 \mathrm{~b}$ & $\mathrm{D}$ & 2,90 & $\mathrm{~cd}$ & $\mathrm{C}$ & $3,45 \mathrm{~b}$ & B & 3,88 & bcd & $\mathrm{B}$ & 4,55 abcd & A \\
\hline dimetomorfe+ametoctradinaa & $1,00 \mathrm{~b}$ & $\mathrm{D}$ & 2,53 & def & $\mathrm{C}$ & 2,80 bcde & $\mathrm{BC}$ & 3,53 & cdef & $\mathrm{B}$ & $4,35 \mathrm{abcd}$ & A \\
\hline dimetomorfe + mancozebe & $1,00 \mathrm{~b}$ & $\mathrm{D}$ & 2,93 & $\mathrm{~cd}$ & $\mathrm{C}$ & $3,48 \mathrm{~b}$ & $\mathrm{~B}$ & 3,90 & bcd & $\mathrm{B}$ & $4,58 \mathrm{abc}$ & A \\
\hline dimetomorfe + clorotalonil & $1,00 \mathrm{~b}$ & $\mathrm{D}$ & 2,78 & de & $\mathrm{C}$ & $3,25 \mathrm{~b}$ & B & 3,70 & cdef & $\mathrm{B}$ & $4,50 \mathrm{abcd}$ & A \\
\hline cimoxanil + mancozebe & $1,00 \mathrm{~b}$ & $\mathrm{E}$ & 2,48 & def & $\mathrm{D}$ & $3,20 \mathrm{bc}$ & $\mathrm{C}$ & 4,10 & $\mathrm{bc}$ & $\mathrm{B}$ & $4,83 \mathrm{ab}$ & A \\
\hline cimoxanil + zoxamida & $1,00 \mathrm{~b}$ & $\mathrm{E}$ & 2,50 & def & $\mathrm{D}$ & $3,25 \mathrm{~b}$ & $\mathrm{C}$ & 3,75 & bcde & $\mathrm{B}$ & $4,50 \mathrm{abcd}$ & A \\
\hline mefenoxam + mancozebe & $1,00 \mathrm{~b}$ & $\mathrm{D}$ & 2,30 & efg & $\mathrm{C}$ & 2,68 bcde & $\mathrm{BC}$ & 2,88 & gh & $\mathrm{B}$ & 3,88 & A \\
\hline mefenoxam + clorotalonil & $1,00 \mathrm{~b}$ & $\mathrm{D}$ & 1,85 & $g$ & $\mathrm{C}$ & 2,13 & $\mathrm{C}$ & 2,95 & gh & $\mathrm{B}$ & 4,00 & A \\
\hline bentiavalicarbe + fluazinam & $1,00 \mathrm{~b}$ & $\mathrm{E}$ & 2,05 & $\mathrm{fg}$ & $\mathrm{D}$ & 2,60 & $\mathrm{C}$ & 3,15 & fgh & B & 4,08 & A \\
\hline mandipropamida & $1,00 \mathrm{~b}$ & $\mathrm{E}$ & 2,50 & def & $\mathrm{D}$ & 3,08 bcd & C & 3,83 & bcd & $\mathrm{B}$ & 4,40 bcde & A \\
\hline mandipropamida+clorotalonil & $1,00 \mathrm{~b}$ & $\mathrm{E}$ & 2,33 & defg & $\mathrm{D}$ & $2,85 \mathrm{bcd}$ & C & 3,55 & cdef & B & 4,28 bcde & A \\
\hline famoxadona + cimoxanil & $1,00 \mathrm{~b}$ & $\mathrm{E}$ & 2,13 & fg & $\mathrm{D}$ & 2,65 cde & $\mathrm{C}$ & 4,00 & bcd & $\mathrm{B}$ & 4,50 abcd & A \\
\hline fenamidona & $1,00 \mathrm{~b}$ & $\mathrm{E}$ & 2,58 & def & $\mathrm{D}$ & $3,18 \mathrm{bc}$ & $\mathrm{C}$ & 4,30 & $\mathrm{~b}$ & B & $4,78 \mathrm{ab}$ & A \\
\hline propamocarbe & $1,00 \mathrm{~b}$ & $\mathrm{D}$ & 2,25 & efg & $\mathrm{C}$ & 2,68 bcde & C & 3,30 & defgh & B & 4,14 cdef & A \\
\hline fenamidona + propamocarbe & $1,00 \mathrm{~b}$ & $\mathrm{D}$ & 2,18 & fg & $\mathrm{C}$ & 2,53 & $\mathrm{C}$ & 3,18 & efgh & B & 3,95 & A \\
\hline fluopicolide + propamocarbe & $1,00 \mathrm{~b}$ & $\mathrm{D}$ & 2,10 & fg & $\mathrm{C}$ & $\begin{array}{l}2,45 \\
\mathrm{BC}\end{array}$ & & 2,63 & $\mathrm{~h}$ & $\mathrm{~B}$ & 4,10 & A \\
\hline fluazinam & $1,55 \mathrm{~b}$ & $\mathrm{C}$ & $3,75 \mathrm{k}$ & & B & $4,80 \mathrm{a}$ & A & 5,00 & & A & 5,00 a & A \\
\hline ciazofamida & $1,25 \mathrm{~b}$ & $\mathrm{C}$ & $3,45 \mathrm{k}$ & bc & B & $4,85 \mathrm{a}$ & A & 5,00 & & A & $5,00 \mathrm{a}$ & $\mathrm{A}$ \\
\hline
\end{tabular}

*Médias seguidas por letras minúsculas na coluna e maiúsculas na linha não diferem entre si pelo teste de Tukey a $5 \%$ de probabilidade.

Tabela 3 - Número de esporângios de Phytophthora infestans em $10 \mu \mathrm{L}$, obtidos em folíolos de batata do cultivar Ágata tratadas com fungicidas 1, 12, 2436 e 48 horas após a inoculação (HAI).

\begin{tabular}{|c|c|c|c|c|c|c|c|c|c|}
\hline \multirow{3}{*}{$\begin{array}{l}\text { Tratamentos } \\
\text { testemunha }\end{array}$} & \multicolumn{9}{|c|}{ Horas após a inoculação (HAI) } \\
\hline & \multicolumn{2}{|l|}{1} & \multicolumn{2}{|c|}{12} & 24 & \multicolumn{2}{|c|}{36} & \multicolumn{2}{|r|}{48} \\
\hline & 326,75 & a $\mathrm{A}$ & $335,25 a$ & A $346,25 a$ & $\mathrm{~A}$ & $360,25 a$ & $\mathrm{~A}$ & $336,25 \mathrm{a}$ & $\mathrm{A}$ \\
\hline piraclostrobina + metiram & 0,00 & $\mathrm{~b} C$ & 18,25 & d BC 40,25 & C B & 80,50 & de $A$ & 112,75 & defg A \\
\hline dimetomorfe + ametoctradina & 0,00 & $\mathrm{~b} C$ & 18,75 & d BC 31,75 & c $\mathrm{BC}$ & 48,75 & efgAB & 72,00 & ghi A \\
\hline dimetomorfe + mancozebe & 0,00 & $\mathrm{~b} C$ & 13,00 & d C 53,00 & C B & 105,50 & A & 140,50 & cde \\
\hline dimetomorfe + clor & 0,00 & b D & 25,00 & cd CD 42,25 & c $\mathrm{C}$ & 86,00 & de & 126,75 & cdef \\
\hline cimoxanil + mancozebe & 0,00 & b D & 36,75 & bcd CD 57,50 & c $\mathrm{BC}$ & 94,25 & de & 156,75 & $\mathrm{~cd}$ \\
\hline cimoxanil + & 0,00 & b D & 23,00 & cd CD 50,50 & c $\mathrm{BC}$ & 85,50 & de $A B$ & 118,00 & cdefg A \\
\hline mefe & 0,00 & $\mathrm{~b} B$ & 8,50 & d $A B \quad 15,25$ & c $\mathrm{AB}$ & 28,25 & gAB & 39,50 & ijA \\
\hline mefe & 0,00 & b A & 5,75 & d A 11,25 & C A & 20,25 & gA & 22,50 & j A \\
\hline zinam & 0,00 & b D & 26,00 & cd CD 46,25 & c $\mathrm{BC}$ & 81,25 & de $A B$ & 114,00 & defgh $\mathrm{A}$ \\
\hline man & 0,00 & b D & 18,75 & d CD 43,75 & c $\mathrm{BC}$ & 66,25 & $\operatorname{defg} A B$ & 91,25 & fgh $A$ \\
\hline mandipropamida+clorotalonil & 0,00 & $\mathrm{~b} C$ & 12,25 & d BC 33,75 & c $\mathrm{ABC}$ & 48,25 & efg $A B$ & 68,25 & hijA \\
\hline famoxadona + cimoxanil & 0,00 & b D & 20,50 & cd CD 53,50 & c C & 103,50 & d B & 163,50 & A \\
\hline fenamidona & 0,00 & $\mathrm{~b} D$ & 20,05 & cd CD 46,75 & c $\mathrm{BC}$ & 76,75 & def $A B$ & 105,50 & efgh A \\
\hline propamocarbe & 0,00 & $\mathrm{~b} C$ & 19,75 & cd BC 52,25 & C B & 92,25 & de & 117,25 & cdefg \\
\hline fenamidona + propamocarbe & 0,00 & $\mathrm{~b} C$ & 13,75 & d C 31,50 & c $\mathrm{BC}$ & 51,50 & efg B & 76,50 & ghi A \\
\hline fluopicolide + propamocarbe & 0,00 & $\mathrm{~b} B$ & 12,75 & d $\mathrm{AB} 17,25$ & c $\mathrm{AB}$ & 29,00 & $\mathrm{fg} B$ & 41,50 & \\
\hline fluazinam & 0,00 & b D & 80,05 & C 162,50 & B & 270,25 & $\mathrm{~A}$ & 297,75 a & A \\
\hline ciazofamida & 0,00 & b D & 67,75 & C 124,25 & B & 200,55 & A & $217,75 \mathrm{~b}$ & A \\
\hline
\end{tabular}

*Médias seguidas por letras minúsculas na coluna e maiúsculas na linha não diferem entre si pelo teste de Tukey a $5 \%$ de probabilidade. 


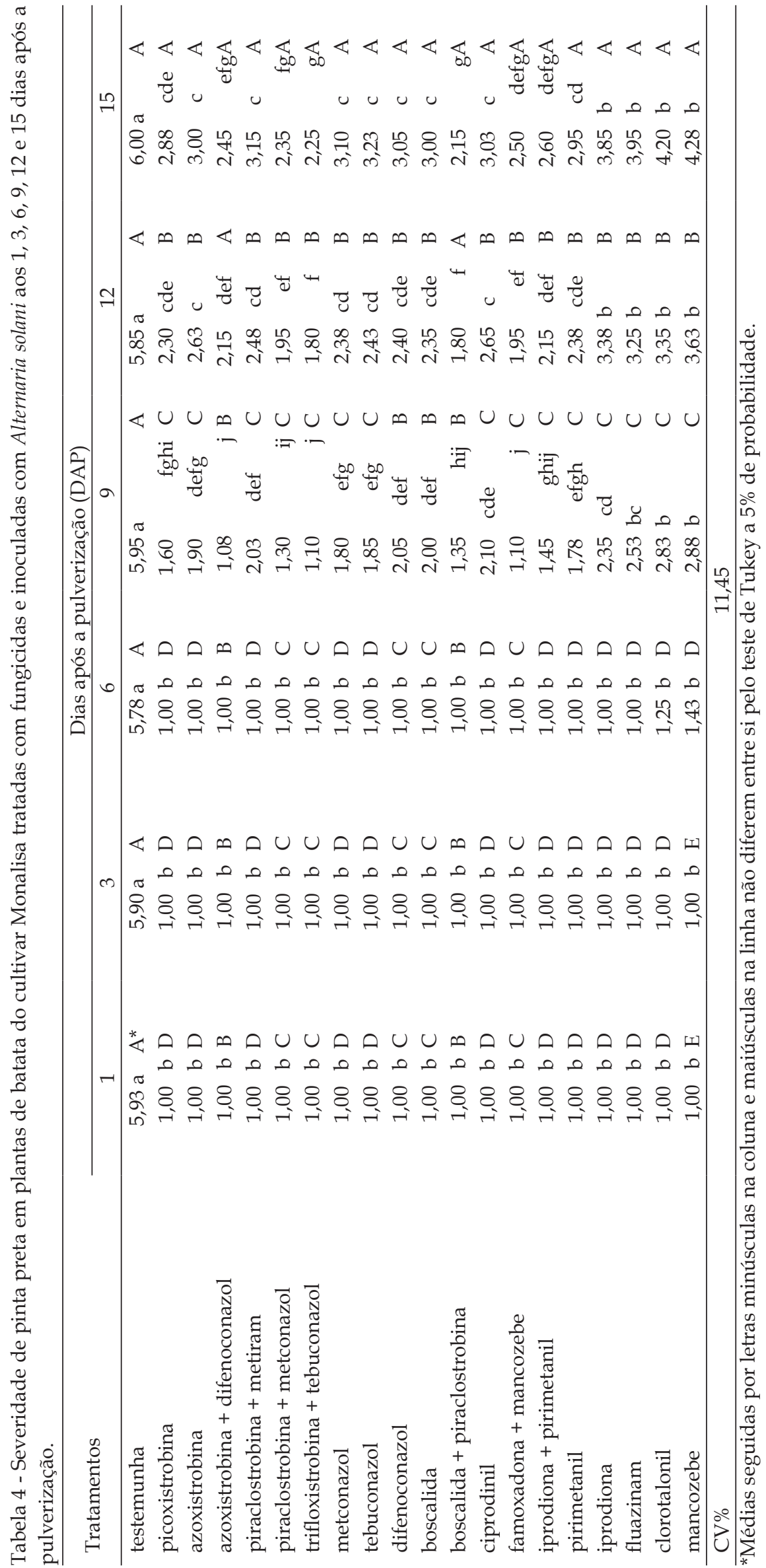


Tabela 5 - Severidade da pinta preta em plantas de batata do cultivar Monalisa inoculadas com Alternaria solani e tratadas com fungicidas: uma, 24, 48 e 72 horas após a inoculação (HAI).

\begin{tabular}{|c|c|c|c|c|c|c|c|c|}
\hline \multirow{3}{*}{$\begin{array}{l}\text { Tratamentos } \\
\text { picoxistrobina }\end{array}$} & \multicolumn{8}{|c|}{ Horas após a inoculação (HAI) } \\
\hline & 1 & \multicolumn{3}{|c|}{24} & \multicolumn{2}{|l|}{48} & \multicolumn{2}{|l|}{72} \\
\hline & $1,00 \quad b \quad D^{*}$ & 1,95 & efg & $\mathrm{C}$ & 3,40 & B & 4,10 & $\mathrm{~A}$ \\
\hline azoxistrobina & 1,00 b D & 2,43 & cdef & $\mathrm{C}$ & 3,88 defg & $\mathrm{B}$ & 4,50 cdefg & A \\
\hline azoxistrobina + difenoconazol & 1,00 b D & 1,80 & $\mathrm{fg}$ & $\mathrm{C}$ & 3,23 & $\mathrm{~B}$ & 3,78 & A \\
\hline piraclostrobina + metiram & 1,00 b D & 2,45 & cdef & $\mathrm{C}$ & 4,30 cde & B & $4,90 \mathrm{bcd}$ & A \\
\hline piraclostrobina + metconazol & 1,00 b C & 2,08 & efg & $\mathrm{B}$ & 3,43 & A & 3,85 & A \\
\hline trifloxistrobina + tebuconazol & $1,00 \mathrm{~b} D$ & 1,60 & $\mathrm{~g}$ & $\mathrm{C}$ & 2,95 & B & 3,55 & A \\
\hline tebuconazol & $1,00 \mathrm{~b} \quad \mathrm{D}$ & 2,23 & defg & $\mathrm{C}$ & efgh & B & 4,15 & A \\
\hline metconazol & 1,00 b D & 2,28 & defg & C & 3,38 & $\mathrm{~B}$ & defgh & A \\
\hline difenoconazol & $1,00 \mathrm{~b} \quad \mathrm{D}$ & 2,13 & defg & $\mathrm{C}$ & 3,68 efgh & B & 4,28 cdefgh & A \\
\hline boscalida & 1,00 b C & 2,40 & cdef & B & 4,28 cdef & A & 4,60 cde & $\mathrm{A}$ \\
\hline boscalida+piraclostrobina & 1,00 b C & 2,03 & efg & B & 3,73 efg & A & 4,10 & A \\
\hline ciprodinil & $1,00 \mathrm{~b} D$ & 2,60 & cde & $\mathrm{C}$ & 3,53 fgh & B & 4,35 cdefg & A \\
\hline famoxadona+mancozebe & $1,00 \mathrm{~b} \mathrm{C}$ & 3,10 & $\mathrm{bc}$ & $\mathrm{B}$ & $4,50 \mathrm{~cd}$ & A & $4,95 \mathrm{bc}$ & $\mathrm{A}$ \\
\hline fluazinam & $1,00 \mathrm{~b} \mathrm{C}$ & 3,63 & $\mathrm{~b}$ & B & $5,25 \mathrm{ab}$ & A & $5,60 \mathrm{ab}$ & $\mathrm{A}$ \\
\hline iprodiona+pirimetanil & $1,00 \mathrm{~b} D$ & 2,38 & cdef & C & 3,75 efg & B & 4,53 cdef & A \\
\hline iprodiona & 1,00 b D & 2,83 & $\mathrm{~cd}$ & C & $4,63 \mathrm{bc}$ & B & $5,70 \mathrm{a}$ & A \\
\hline pirimetanil & 1,00 b D & 2,45 & cdef & $\mathrm{C}$ & 3,83 defg & B & 4,43 cdefg & A \\
\hline testemunha & 5,85 a $\mathrm{A}$ & 5,75 & $\mathrm{a}$ & $\mathrm{A}$ & $5,73 \mathrm{a}$ & $\mathrm{A}$ & $5,95 \mathrm{a}$ & A \\
\hline $\mathrm{CV} \%$ & & & & & 5,38 & & & \\
\hline
\end{tabular}

*Médias seguidas por letras minúsculas na coluna e maiúsculas na linha não diferem entre si pelo teste de Tukey a 5\% de probabilidade.

Tabela 6 - Número de conídios de Alternaria solani em $10 \mu \mathrm{L}$, obtidos em folíolos de batata do cultivar Monalisa tratados com fungicidas, 1, 24, 48 e 72 horas após a inoculação (HAI).

\begin{tabular}{|c|c|c|c|c|c|c|c|}
\hline \multirow{3}{*}{$\begin{array}{l}\text { Tratamentos } \\
\text { testemunha }\end{array}$} & \multicolumn{7}{|c|}{ Horas após a inoculação (HAI) } \\
\hline & \multicolumn{2}{|l|}{1} & \multicolumn{2}{|l|}{24} & \multicolumn{2}{|c|}{48} & 72 \\
\hline & $194,75 \mathrm{a}$ & $\mathrm{AB}$ & $186,50 \mathrm{a}$ & $\mathrm{B}$ & $199,75 \mathrm{a}$ & $\mathrm{AB}$ & $212,75 \mathrm{a}$ \\
\hline picoxistrobina & 0,00 & b C & 17,00 & $\mathrm{BC}$ & 39,00 & B & $85,25 \mathrm{~cd}$ \\
\hline azoxistrobina & 0,00 & b C & 18,75 & $\mathrm{C}$ & 51,50 & $\mathrm{~B}$ & 78,50 cde $A$ \\
\hline azoxistrobina + difenoconazol & 0,00 & b C & 8,25 & C & 32,50 & B & 55,05 ef $\mathrm{A}$ \\
\hline piraclostrobina + metiram & 0,00 & b D & 27,00 def & C & 54,50 cde & B & 88,75 cd A \\
\hline piraclostrobina + metconazol & 0,00 & $\mathrm{~b} \quad \mathrm{C}$ & 10,75 & $\mathrm{BC}$ & 23,25 & $\mathrm{AB}$ & 42,00 \\
\hline trifloxistrobina + tebuconazol & 0,00 & b C & 11,75 & $\mathrm{BC}$ & 31,00 & B & 53,50 \\
\hline tebuconazol & 0,00 & b D & 27,25 def & $\mathrm{C}$ & 49,75 & $\mathrm{~B}$ & 77,25 cde A \\
\hline metconazol & 0,00 & b C & 33,75 & B & 46,75 & B & 78,75 cde $A$ \\
\hline difenoconazol & 0,00 & b C & 33,05 def & B & 59,00 cde & A & 80,75 cde A \\
\hline boscalida & 0,00 & b C & 35,15 def & B & 51,25 & B & 78,75 cde A \\
\hline boscalida + piraclostrobina & 0,00 & b C & 11,05 & $\mathrm{BC}$ & 29,05 & $\mathrm{AB}$ & 51,50 \\
\hline ciprodinil & 0,00 & $\mathrm{~b} \quad \mathrm{D}$ & 25,25 def & $\mathrm{C}$ & 55,75 cde & B & 78,25 cde $A$ \\
\hline famoxadona + mancozebe & 0,00 & b C & $52,50 \mathrm{bcd}$ & $\mathrm{B}$ & $81,05 \mathrm{bcd}$ & A & 91,00 cd A \\
\hline fluazinam & 0,00 & b D & $75,50 \mathrm{~b}$ & $\mathrm{C}$ & $103,00 \mathrm{~b}$ & B & $150,50 \mathrm{~b}$ \\
\hline iprodiona + pirimetanil & 0,00 & b C & 24,75 def & B & 44,25 & $\mathrm{AB}$ & 63,25 \\
\hline iprodiona & 0,00 & $\mathrm{~b} \quad \mathrm{D}$ & $65,50 \mathrm{bc}$ & $\mathrm{C}$ & $105,50 \mathrm{~b}$ & B & $133,50 \mathrm{~b}$ \\
\hline pirimetanil & 0,00 & b C & 41,00 cde & $\mathrm{B}$ & 56,05 cde & A & 98,25 \\
\hline $\mathrm{CV} \%$ & & & & & & & \\
\hline
\end{tabular}

*Médias seguidas por letras minúsculas na coluna e maiúsculas na linha não diferem entre si pelo teste de Tukey a 5\% de probabilidade. 
No experimento 4, não foram observados sintomas da pinta preta nas plantas tratadas no intervalo de 1 HAI (Tabela 5). Nos intervalos de 24, 48 e 72 HAI, os menores níveis da doença foram obtidos nas plantas tratadas com picoxistrobina, azoxistrobina + difenoconazol, piraclostrobina + metconazol, trifloxistrobina + tebuconazol, tebuconazol, difenoconazol, metconazol e boscalida + piraclostrobina. Azoxistrobina, boscalida, ciprodinil, famoxadona + mancozebe, iprodiona + pirimetanil e pirimetanil apresentaram comportamento intermediário. Fluazinam e iprodiona foram os menos efetivos, não diferindo da testemunha no último intervalo. Os maiores níveis de ação curativa foram verificados quando os tratamentos foram aplicados até $24 \mathrm{HAI}$. Osfungicidas testados reduziram significativamente a esporulação de $A$. solani em todos os intervalos testados (Tabela 6). Os maiores níveis de atividade antiesporulanteforam observados para os fungicidas com maior ação curativa. O aumento do intervalo entre a inoculação e a aplicação foi significativo sobre a atividade anti esporulante dos fungicidas. Os menores números de conídios de $A$ solani foram observados nos intervalos de 1 e 24 HAI.

Nesse estudo, todos os fungicidas, independente do seu modo de ação, proporcionaram elevada ação protetora no controle da requeima e da pinta preta da batata. A ação protetora de fungicidas, com diferentes níveis de sistemicidade, no controle dessas doenças também é ressaltada por BODKER; Nielsen (2001), Bodker; Nielsen (2002) e Horsfield et al. (2010).

Os maiores níveis de ação residual foram obtidos com produtos móveis sendo as primeiras reduções de controle observadas a partir dos 12 DAP. Para a requeima, os melhores resultados foram obtidos com as misturas contendo os sistêmicos mefenoxam e propamocarbe ou com produtos com diferentes níveis de mobilidade e alta tenacidade, como mandipropamida, dimetomorfe, ametoctradina, famoxadona e zoxamida. Para a pinta preta destacaram-se principalmente as misturas azoxistrobina + difenoconazol, trifloxistrobina + tebuconazol, piraclostrobina + metconazol e boscalida + piraclostrobina. A ação residual superior dos fungicidas móveis ou com alta tenacidade deve-se, principalmente, a suas respectivas capacidades de serem absorvidas e translocadas pelos tecidos ou por apresentarem maior capacidade de adesão às camadas lipofílicas existentes na superfície das folhas (COHEN; COFFEY, 1986; SAUTER, 2007; KuCK; VORS, 2007; Goldetal., 2009).

Os produtos formulados com cimoxanil e piraclostrobina para requeima e picoxistrobina, azoxistrobina, iprodiona, pirimetanil, iprodiona + pirimetanil, para pinta preta apresentaram as primeiras reduções de controle aos 9DAP. Comportamento semelhante foi observado para boscalida eciprodinil, e produtos decontato como fluazinam eciazofamida. A baixa ou lenta penetração nos tecidos, a menor adesão e a exposição a fatores externos podem justificar a menor ação residual desses produtos em relação aos demais (HEWIT, 1998; BARTLETT et al., 2001; GISI; MÜLLER, 2007). Os períodos de controle da pinta preta obtidos nesse estudo com os fungicidas azoxistrobina, piraclostrobina + metiram, tebuconazol, difenoconazol, boscalida efamoxadona + mancozebe foram inferiores aos obtidos por TöFoli et al. (2005) em tomateiro. Esse resultado pode ser atribuído a diferenças na absorção ou aderência proporcionada pelas superfícies foliares das duas solanáceas, suscetibilidade dos cultivares ou, ainda, diferenças de agressividade dos isolados empregados.

Os menores períodos de controle foram obtidos com mancozebe e clorotalonil para requeima, e mancozebe para pinta preta. Esses fungicidas de contato típicos são expostos de forma contínua a fatores ambientais externos como fotólise, hidrólise, volatilização, ação de chuvas e umidade. A maior ação residual de clorotalonil em relação ao mancozebe sobre a pinta preta pode ser justificada por sua tenacidade e maior fungitoxicidade sobre $A$. solani (Suehi; Latin, 1991).

Os maiores níveis de ação curativa e atividade anti-esporulante no controle da requeima da batata foram obtidos com produtos sistêmicos ou mesostêmicos contendo mefenoxam + mancozebe, mefenoxam + clorotalonil, propamocarbe, fluopicolide + propamocarbe, fenamidona + propamocarbee dimetomorfe + amectoctradina quando aplicados até $24 \mathrm{HAI}$. Os fungicidas translaminares como mandipropamida, cimoxanil, fenamidona e dimetomorfe em mistura com clorotalonil e mancozebe apresentaram comportamento intermediário e limitaram o desenvolvimento da requeima principalmente quando aplicados até 12 HAI. BodKer; Nielsen (2001, 2002) observaram que produtos sistêmicos e translaminares apresentaram ação curativa sobre a requeima principalmente quando aplicados no início do período de latente, ou de 12 a 36 horas após a inoculação. A redução da ação curativa e anti esporulante dos fungicidas, nos intervalos de 36 e $48 \mathrm{HAI}$, pode ser justificada pelo rápido caráter destrutivo da requeima, que dificulta a absorção e a translocação do produto e, consequentemente, a sua ação sobre o patógeno no interior dos tecidos.

Os melhores níveis de ação curativa e anti esporulante para pinta preta foram observados para fungicidas que possuem ação sistêmica ou translaminar como: picoxistrobina, azoxistrobina + difenoconazol, piraclostrobina + metconazol, trifloxistrobina + tebuconazol, tebuconazol, difenoconazol, metconazol, boscalida + piraclostrobina, azoxistrobina eboscalida até o intervalo de 24 HAI (SAUTER, 2007; KUCK; VorS, 2007; RHEINHEIMER, 2007). 
A elevada ação protetora, residual, curativa e anti esporulantedefluopicolide + propamocarbeefenamidona + propamocarbe obtidas nesse estudo podem ser explicadas pela significativa ação translaminar de fluopicolide, mesostêmica defenamidona, esistêmica de propamocarbe. O propamocarbe apresenta a capacidade de penetrar e mover-se pelos tecidos e agir diretamente sobre o crescimento micelial e desenvolvimento de esporângios (JoHNSON et al., 2000). As duas misturas se diferenciam principalmente pelo mecanismo distinto de ação de fluopicolide e fenamidona. Fluopicolide atua interrompendo a formação de proteínas que desempenham papel vital na estabilidade das células em oomicetos (ToQuin et al., 2006), enquanto que a fenamidona age inibindo a respiração ao nível do complexo III, QoI(SAUTER, 2007). O fluopicolide e fenamidona tendem a se distribuir na superfície tratada e promover a formação de uma película protetora. Com o passar do tempo, parte dos depósitos de fluopicolide, existentes na superfície foliar, tende a ser absorvida e distribuída via xilema, enquanto que os de fenamidona são redistribuídos sobre a superfície foliar (TAFFOREAU et al., 2006; TAFFOREAU et al. 2009).

A alta ação protetora e residual observada para mandipropamida e mandipropamida + clorotalonil pode ser explicada pela sua forte aderência às ceras presentes na superfície foliar. Apesar de não ter sido notada diferença na ação residual entre mandipropamida e mandipropamida + clorotalonil é importante destacar que clorotalonil também apresenta reconhecida tenacidade e persistência (SUEHI; LATIN, 1991). Mandipropamida e mandipropamida + clorotalonil apresentaram controle curativo intermediário da requeima. Segundo Huggenberger; KNAUF-BEITER (2007), mandipropamida apresenta boa ação translaminar, porém sua ação curativa e atividade anti esporulante podem ser limitadas. A alta afinidade de mandipropamida pelas ceras superficiais das folhas faz com que a maior parte do produto fique aderida na parte externa, sendo insuficiente para inibir completamente o patógeno no interior dos tecidos.

A elevada ação protetora de bentiavalicarbe + fluazinam verificada nesse trabalho também foi constatada por MiYAKE et al. (2005) em plantas de tomate tratadas com bentiavalicarbe em condições controladas. Segundo Hofman; VAn OudHeusden (2004), bentiavalicarbe apresenta boa estabilidade e ação translaminar, o que permite proteger adequadamente as plantas tratadas. No Brasil, bentiavalicarbe é formulado em mistura com fluazinam, outro fungicida com reconhecida ação residual (Whittingham, 2007). A significativa ação curativa de bentiavalicarbe até as $12 \mathrm{HAI}$, observada nesse estudo, também foi constatada por MiYAKE et al. (2005) em plantas de tomate.
As plantas de batata tratadas com dimetomorfe em mistura com diferentes fungicidas apresentaram diferentes períodos de proteção. Dimetomorfe + ametoctradina e dimetomorfe + clorotalonil foram semelhantes entre si e superiores a mistura dimetomorfe + mancozebe. Considerando que dimetomorfe possui sistemicidade apoplástica moderada (COHEN et al., 1995) conclui-se que a diferenciação da ação residual entre essas misturas deve-se principalmente à elevada estabilidade e afinidade de ametoctradina com as camadas de cera na superfície foliar (Gold et al., 2009; REIMANN et al., 2010) e a considerável tenacidade de clorotalonil, em relação a mancozebe (SueHi; Latin, 1991). A ação curativa de dimetomorfe e suas misturas com mancozebe e clorotalonil até o intervalo de $12 \mathrm{HAI}$ foi igualmente verificada por JoHNSON et al. (2000). A maior ação curativa de dimetomorfe + ametoctradina até o intervalo de $24 \mathrm{HAI}$ e a redução da esporulação de $P$. infestans nos intervalos de 36 e 48 HAI sugerem que ametoctradina possa ter alguma atividade sistêmica, ou a formulação permita que os níveis de penetração de dimetomorfe nos tecidos sejam maiores.

Mefenoxam e as suas misturas com mancozebe e clorotalonil apresentaram elevada ação protetora, residual, curativa e anti esporulante graças às propriedades sistêmicas dessa acilalanina (COHEN; COFFEy, 1986; BodKer; Nielsen, 2001; BOdKER; NIELSEN 2002; Singh; PundHIR, 2004).

A superioridade da ação residual decimoxanil em mistura com zoxamida e famoxadona, em relação ao mancozebe, deve-se principalmente à estabilidade e elevada tenacidade apresentada por zoxamida (BRADSHAW; SCHEPERS, 2001) e famoxadona (ANDRIEU et al., 2001), uma vez que cimoxanil apresenta baixa ação residual (CoHEN; Coffey, 1986) e mancozebe baixa tenacidade(SuEHI; Latin, 1991). Nesse estudo, as misturas com cimoxanil apresentaram considerável ação curativa e redução da esporulação quando aplicadas no intervalo de 12 HAI. JoHnson et al. (2000) também observaram significativa ação curativa de cimoxanil sobre a requeima da batata, quando as plantas foram tratadas até as $24 \mathrm{HAI}$. A menor ação curativa de cimoxanil, observada nesse estudo, pode ser justificada pela alta suscetibilidade do cultivar Ágata à requeima e pelo uso de uma concentração de inóculo superior à utilizada por esses autores. A ação anti esporulante observada para cimoxanil e suas misturas concorda com os resultados de GENET et al. (2000).

Apesar de piraclostrobina apresentar registro no Brasil para o controle da requeima, as demais representantes do grupo como azoxistrobina, trifloxistrobina e picoxistrobina têm seu foco voltado principalmente para o controle da pinta preta, sejam isoladas ou em mistura com triazóis. De maneira 
geral, as estrobilurinas não diferiram entre si quanto à ação residual, porém piraclostrobina foi inferior as demais em relação à ação curativa. Esse resultado pode ser justificado pela menor sistemicidade de piraclostrobina em relação à picoxistrobina, trifloxistrobina e azoxistrobina (BARTLETT et al., 2001; SAUTER, 2007).

A superioridade de boscalida + piraclostrobina, em relação à piraclostrobina, pode ser justificada pela considerável ação translaminar e acropetal da boscalida (RHEINHEIMER, 2007).

Os resultados positivos obtidos com tebuconazol, difenoconazol, metoconazol e flutriafol no controle da pinta preta podem ser explicados pelos diferentes níveis de sistemicidade e alta fungitoxicidade apresentada por esses triazóis (ForCELINI, 1994).

A alta ação residual de misturas como azoxistrobina + tebuconazol, piraclostrobina + metconazol, boscalida + piraclostrobina, em relação ao uso isolado de seus ingredientes ativos, sugere que o somatório de características, como diferentes mecanismos de ação e diferentes níveis de sistemicidade, contribua para uma maior eficácia e maior período de proteção. Cabe destacar que essas misturas não promoveram maior controle curativo em relação ao uso isolado. Esse resultado pode ser explicado pelo fato dos ingredientes ativos em mistura possuírem a mesma solubilidade e grau de penetração nos tecidos dos ingredientes ativos isolados.

A considerável ação curativa dos fungicidas azoxistrobina, piraclostrobina + metiram, boscalida, difenoconazol, tebuconazol e pirimetanil observada nesse trabalho concorda com os obtidos por TöFoliet al. (2005) em plantas de tomate tratadas até 24 horas após a inoculação. A ação curativa de azoxistrobina no controle da pinta preta da batata foi verificada por BOUWMAN; RIJKERS (2004) em plantas de batata tratadas 48 horas após a inoculação. Horsfield et al. (2010) também destacaram a ação curativa de boscalida, azoxistrobina e difenoconazol quando aplicados 3 dias após a inoculação. Fluazinam e iprodiona promoveram redução da pinta preta somente quando aplicados até $24 \mathrm{HAI}$, porém, em nível inferior aos demais fungicidas. A limitada ação curativa desses fungicidas no controle da pinta preta do tomateiro também foi observada por KомYoJI et al. (1995) e TöFoli et al. (2005). Existem poucas informações disponíveis sobre a atividade anti esporulante de fungicidas sobre A solani. Bouwan; RIJKERS (2004) obtiveram, em condições controladas, excelente e intermediária redução da esporulação desse fungo para azoxistrobina e difenoconazol, respectivamente. TöFoli et al. (2005) observaram considerável ação anti esporulante dos fungicidas azoxistrobina, cresoxim metílico, difenoconazol, tebuconazol, pirimetanil em plantas de tomate tratadas pré e pós-inoculação.
A elevada ação residual e a limitada ação curativa de famoxadona e suas misturas observadas nesse trabalho pode ser justificada por sua elevada tenacidade e baixo potencial de translocação (ANDRIEU et al., 2001; SAUTER, 2007).

Nesse estudo, iprodiona, pertencente ao grupo das dicarboximidas, apresentou níveis intermediários de ação residual, curativa e anti esporulante sobre a pinta preta. Tal resultado pode ser explicado por sua baixa penetração nos tecidos (HewiTT, 1998). A superioridade da ação curativa e anti esporulante de iprodiona + pirimetanil pode serjustificada pela maior sistemicidade do pirimetanil (GISI; MÜLLER, 2007).

Os fungicidas pirimetanil e ciprodinil, pertencentes ao grupo das anilinopirimidinas, também promoveram níveis intermediários de ação residual, curativa e anti esporulante. Pirimetanil atua inibindo a secreção de proteínas e enzimas associadas com a patogênese, enquanto que o ciprodinil inibe a síntese de aminoácidos intermediários. O modo distinto de ação desses fungicidas os torna importantes ferramentas em programas de manejo (GISI; MüLLER, 2007).

Os resultados desse estudo enfatizam que os melhores resultados de controle das duas doenças foram obtidos quando esses são aplicados de forma preventiva. A ação curativa é uma característica importante e desejável, porém, na prática é difícil definir o momento oportuno da aplicação no campo. Em situações específicas em que existe a necessidade do uso curativo de fungicidas deve-se optar sempre por misturas que incluam produtos sistêmicos e com diferentes mecanismos de ação. Destaca-se ainda que aplicações curativas em situações de grande pressão de doença, além de pouco eficazes, podem selecionar raças resistentes do patógeno (GHINI; KimATI, 2000). A redução da esporulação de $P$. infestans e $A$. solani observada nesse estudo pode refletir de forma significativa na redução do inóculo e progresso dessas doenças no campo.

\section{CONCLUSÃO}

Concluiu-se que todos os fungicidas, independente de seu modo de ação, promoveram elevada proteção contra a requeima e a pinta preta da batata em condições controladas. Os fungicidas sistêmicos apresentaram redução de controle a partir dos 12 DAP, enquanto que fungicidas translaminares e mesostêmicos a partir dos 9 DAP. Quanto à ação curativa e anti esporulante destacaram-se principalmente os fungicidas sistêmicos aplicados até as 24 horas após a inoculação (HAI). Para requeima, alguns fungicidas translaminares e mesostemicos foram capazes de inibir a doença quando aplicados até $12 \mathrm{HAI}$. Os fungicidas de contato destacaram-se apenas para a característica ação protetora. 


\section{REFERÊNCIAS}

AGRIOS, G.N. Plant pathology. 5.ed. Amsterdam Elsevier Academic Press, 2005. 919p.

ANDRIEU, N.; JAWORSKA, G.; GENET J.; BOMPEIX, G. Biological mode of action of Famoxadone on Plasmopara viticola and Phytophthora infestans. Crop Protection, v.20, n.3, p.253-260, 2001.

AZEVEDO, L.A.S. Fungicidas sistêmicos: prática e teoria. Campinas: O Autor, 2007. 290p.

BAIN, R.A. Report of the fungicide sub-group: Discussion of potato late blight fungicides, their properties and ratings. PPO-Special Report, v.13, p.177-182, 2009.

BARTLETT, D.W.; CLOUGH J.M.; GODFREY, C.R.A.; GODWIN, J.R.; HALL, A.A.; HEANEY S.P.; MAUND S.J. Understanding the strobilurin fungicides. Pesticide Outlook, v.12, p.143-148, 2001.

BODKER, L.; NIELSEN, B.J. Preventive and curative effect of fungicides against potato late blight under field conditions. PPV-Special Report, v.7, p.261-264, 2001.

BODKER, L.; NIELSEN, B.J. Field Experiments with preventive and curative control of potato late blight PPO-Special Report, v.8, p.211-215, 2002.

BOUWMAN, J.J.; RIJKERS, G. The control of Alternaria solani (early blight) with azoxystrobin in potatoes. $P P O$ Special Report, v.10, p.179-188, 2004.

BRADSHAW, N.J.; SCHEPERS, H.T.A.M. Experiences with RH-117281 (zoxamide) - a new fungicide for the control of potato blight. PPV-Special Report, v. 7, p. 173183,2001

COHEN, Y.; COFFEY M. D. Systemic fungicides and the control of oomycetes. Annual Review Phytopathology, v.24, p.311-338, 1986.

COHEN, Y.; BALDER, A.; COHEN, B.H. Dimetomorph activity against oomycete fungal plant pathogens. Phytopathology, v.85, p.1500-1506, 1995.

FORCELINI, C.A. Fungicidas inibidores da síntese de esteróis. I Triazoles. Revisão Anual de Patologia de Plantas, v.2, p.335-355, 1994.

GENET, J.L.; JAWORSKA, G.; GEDDENS, R.; SHEPERD, C. Effect of temperature on the curative and anti-sporulant action of cymoxanil for control of Phytophthora infestans. Fifth Workshop of an European Network for development of an Integrated Control Strategy of potato late blight, Munich, p.107-117, 2000.

GHINI, R.; KIMATI, H. Resistência de fungos a fungicidas. Jaguariúna: Embrapa - Meio Ambiente, 2000.

78p.
GISI, U.; MÜLLER, U. Anilinopyrimidines: Methionine Biosynthesis Inhibitors. In: KRÄMER, W.; SCHIRMER, U. (Ed.). Modern Crop Protection Compounds, v.2, p.551560, 2007.

GOLD, R.; SCHERER, M.; RETHER J.; SPEAKMAN J.; NAVÉ, B.; LEVY T.; STORER R.; MARRIS, D. Initium ${ }^{\circledR}$ - An Innovative Fungicide of a New Chemical Class for the Control of Oomycetes. BCPC Congress, 2009.

HERMANN, D.; BARLETT, D.W.; FISCHER, W.; KEMPF, H.J. The behavior of mandipropamid in plants. In: BCPC CROP SCIENCE \& TECHONOLOGY, 2005, Glasgow. Proceedings. Glasgow: BCPC, 2005. p. 93-98.

HEWITT, H.G. Fungicides in Crop Protection. Wallingford: CAB International, 1998. 221p.

HOFMAN, T.W.; VAN OUDHEUSEN, Z. New fungicide benthiavalicarb-isopropil + mancozebe for foliar use in potatoes in Europe. PPO-Special Report, v.10, p.125-30, 2004.

HORSFIELD, A.; WICKS, T.; DAVIES, K.; WILSON, D.; PATON, S. Effect of fungicide use strategies on the control of early blight (Alternaria solani) and potato yield. Australasian Plant Pathology, v.39, n.1, p.368-375, 2010.

HUGGENBERGER, F.; KNAUF-BEITER, G. Mandipropamid a new fungicides for the control of late blight in potatoes. PPO-Special Report, v.12, p. 67-76, 2007.

JAMES, C. An illustrated series of assessment keys for plant diseases, their preparation and usage. Canadian Plant Disease Survey, v.51, p.39-65, 1971.

JOHNSON, D.A.; CUMMINGS, T.F.; GEARY, B. Postinfection activity of selected late blight fungicides. Plant Disease, v.84 n.10, p.1116 - 1120, 2000.

KOMYOJI, T.; SUGIMOTO, K.; MITANI, S.; MATSUO, N.; KAZUMI, S. Biological properties of a new fungicide, fluazinam. Journal Pesticide Science, v.20, p.129-135, 1995.

KUCK K.H.; VORS, J.P. Sterol Biosynthesis Inhibitors. In: KRÄMER, W.; SCHIRMER, U. (Ed.). Modern Crop Protection Compounds, v.2, p.605-650, 2007.

LATORSE, M.P.; JARRY, F.; MERCIER, V.; VELOSO, S. Tuber blight control: effect of foliar applied fungicides such as Infinito on viability of sporangia of Phytophthora infestans. PPO-Special Report, v.12, p.37-47, 2007.

MANTECÓN, J.D. Control of potato early blight with triazole fungicide using preventive and curative spraying, or a forecasting system. Ciencia e Investigación Agraria, v.36, n.2, p.291-296, 2009.

MITAME, S.; KAMACHI, K.; SUGIMOTO, K. Controle of potato late blight by ciazofamid. Journal of Pesticide Science, v.30, n.2, p.116-119, 2005. 
MIYAKE Y.; SAKAI, J.; SHIBATA, M.; YONEKURA, N.; MIURA, I.; KUMAKURA, K.; NAGAYAMA, K.

Fungicidal activity of Benthiavalicarb-isopropyl against Phytophthora infestans and its controlling activity against late blight diseases. Journal Pesticide Science, v.30, n.4, p.390-396, 2005.

REIFSCHNEIDER, F.F.B.; FURUMOTO, O.; FILGUEIRA, F.A.R. Illustrated key for the evaluation of early blight of potatoes. FAO Plant Protection, v.32, n.3, p.9193, 1984

REIMANN, S.; JILDERDA, K.; GERBER, M.; TEGGE, V.; KLAPPACH, K. Initium $®$ : a new fungicidal active ingredient for the control of Oomycetes. PPO-Special Report, v.14, p.89-94, 2010.

REIS, E.M.; REIS A.C.; FORCELINI, C.A. Manual de fungicidas - guia para o controle químico de doenças. Passo Fundo: Ed. Universidade de Passo Fundo, 2007. 157p.

RHEINHEIMER, J. Succinate Dehydrogenase Inhibitors. In: KRÄMER, W.; SCHIRMER, U. (Ed.). Modern Crop Protection Compounds, v.2, p.496-504, 2007.

RUSSEL, P.E. A century of fungicides evolution. Journal of Agricultural Science, v.143, p.11-25, 2005.

SAUTER, H. Strobilurins and other complex III Inhibitors. In: KRÄMER, W.; SCHIRMER, U. (Ed.). Modern Crop Protection Compounds, v.2, p.457-495, 2007.

SINGH, R.P.; PUNDHIR, V.S. Uptake, translocation and persistence of metalaxyl in potato. Journal of Mycology and Plant Pathology, v.34, n.1, p.93-95, 2004.

SUEHI, B.; LATIN, C. Retention of fungicides for control of alternaria leaf spot of muskmelon under greenhouse conditions. Plant Disease, v.75, p.1013-5, 1991.
TAFFOREAU, S.; BARDSLEY, E.; LATORSE, M.P.; FABRÈGES, C.; SCHIRRINGS, A.; WEGMANN, T. Infinito: Profile of a novel potato late blight fungicides. Summary of three years of development trials in Europe. PPO-Special Report, v.11, p.77-88, 2006.

TAFFOREAU, S.; LATORSE, M.P.; JUHL, O.; BARDSLEY, E. Consento: New experiences on the control of late blight 2007-2008. A summary of recent data with Consento in Europe. PPO-Special Report, v.13, p.135-142, 2009.

TOFOLI, J.G. Batata: queima interrompida. Cultivar HF, v.37, p.10-13, 2006.

TOFOLI, J.G.; DOMINGUES, R. J.; KUROZAWA, C. Ação de fungicidas sobre diferentes fases do ciclo da pinta preta do tomateiro em condições controladas. Summa Phytopathologica, v.31, n.2, p.125-132, 2005.

THOMPSON J.M.; COOKE, L.R. The effect of mandipropamid on infection of potato leaves by Phytophthora infestans: an SEM study. PPO-Special Report, v.13, p.229236, 2009.

TOQUIN, V.; BARJA, F.; SIRVEN, C.; GAMET, S.; LATORSE, M.P.; ZUNDEL, L.; SCHMITT, F.; BEFFA, R. A new mode of action for fluopicolid: modification of the cellular localization of a spectrin-like protein. Pflanzenschutz Nachriten Bayer, v.59, p.171-184, 2006.

WHITTINGHAM, W.G. Uncouplers of oxidative phosforylation. In: KRÄMER, W.; SCHIRMER, U. (Ed.). Modern Crop Protection Compounds, v.2, p.505-527, 2007.

Recebido em 12/12/10

Aceito em 15/5/12 